\title{
Estimation des pertes à la récolte du colza d'hiver (Brassica napus) dans des conditions variées
}

\author{
Christophe SAUSSE \\ Dominique WAGNER \\ Jean-Louis LUCAS \\ André ESTRAGNAT \\ Olivier MANGENOT \\ Bernard GARRIC \\ Raymond REAU \\ Céline DEVAUX \\ Josiane CHAMPOLIVIER \\ Antoine MESSÉAN
}

Cetiom, Centre de Grignon, B.P. 4,

78850 Thiverval Grignon,

Fax : 0140799590

<sausse@cetiom.fr>

\begin{abstract}
Oilseed rape seed loss during harvest can cause subsequent unwanted gene flow and thus give raise to management difficulties in order to keep segmented different productions. Furthermore, seed loss reduces benefits of producers. This loss was evaluated in different conditions and trials carried out in three French regions from 2002 to 2004. Seed loss was divided into three categories: before harvest, under the cutter bar and behind the combine harvester. Total loss represented on average $8,2 \%$ of the harvest for grain water content under $12 \%$. But this figure does not take an important variability into account. Indeed, an advanced cutter bar could reduce loss by $80 \%$ and grain humidity at harvest plays an important part in the total loss level and its distribution. Before harvest low grain water content could make seed loss more sensitive to climatic events. Under the cutter bar, seed loss is inversely proportional to grain water content. On the contrary, it increases with grain water content behind the combine, due to loss in green siliquae. These studies can help improving advice to producers and contribute to improve gene flow models.
\end{abstract}

Key words: Oilseed rape, harvesting, seed loss, gene flow

\section{Introduction}

Le colza est une plante déhiscente dont les graines peuvent tomber au sol avant ou pendant la récolte. Ces pertes retiennent aujourd'hui l'attention, puisqu'elles peuvent gêner la coexistence de filières de qualité différenciées, en particulier entre variétés OGM et non OGM [1-3]. En effet, les graines perdues lors d'une récolte de colza peuvent persister plusieurs années [2]. Ces graines peuvent produire des repousses dont les graines et le pollen viendront altérer la qualité du colza suivant, dans le cas où l'agriculteur alterne deux types de colza (par exemple OGM puis conventionnel) sur la même parcelle. D'une manière plus générale, ces repousses peuvent constituer des relais pour des flux de gènes à l'échelle du paysage agricole. D'autre part, des repousses tolérantes à un herbicide total peuvent être difficiles à contrôler dans la rotation, surtout si elles ont acquis des résistances multiples comme cela a déjà été observé au Canada [4].

Cette préoccupation liée à l'impact des OGM ne doit pas faire oublier que les premiers travaux sur les pertes à la récolte réalisés en Angleterre et en Europe de l'Est [5-8] relevaient d'une problématique autre, mais qui garde toute son actualité : les pertes sont avant tout économiques pour le producteur, et il convient d'informer celui-ci des techniques de récolte minimisant ce risque.

Les pertes des graines peuvent survenir à trois niveaux : avant la récolte par égrenage naturel, pendant la récolte sous la barre de coupe et à l'arrière de la moissonneuse après battage. Différentes hypothèses existent sur les facteurs de variation de ces pertes (figure 1), et il apparaît que ces facteurs ne sont pas indépendants: par exemple, le choix d'une date de récolte est lié à l'observation d'un paramètre physiologique (maturité), lui-même lié aux évènements climatiques. Les travaux de recherche sur ce sujet ont principalement porté sur l'estimation de l'effet de certaines pratiques de récolte. L'évaluation des pertes se heurte à des difficultés méthodologiques. La simple comparaison de rendements pour déterminer l'efficacité de certains modes de récolte ne permet pas d'évaluer les pertes. Deux méthodes d'évaluation directe ont été mises au point : Price et al. [3] repris par Hobson et Bruce [9] récupèrent les graines dans des bacs avant la récolte et sous la barre de coupe, mais ne comptabilisent pas les pertes à l'arrière de la machine. Gulden et Shirtliffe [4] mesurent des pertes totales en aspirant puis en triant l'ensemble des résidus laissés après la récolte. Le tableau 1 présente les principales références expérimentales. Les pertes sont variables et peuvent dépendre de facteurs liés au contexte climatique : récolte directe ou après andainage ; variétés de printemps ou d'hiver ; utilisation éventuelle de dessicant.

Ces études ont été réalisées dans des conditions agroécologiques éloignées de celles prévalant en France. Le Cetiom a donc engagé un programme d'évaluation des pertes et de leurs principaux facteurs de variations dans des conditions représentatives de trois bassins de production français. II s'agit d'une démarche davantage exploratoire qu'analytique, dans la mesure où la capacité à tester des facteurs individuellement a été sacrifiée au profit d'une meilleure compréhension du phéno- 


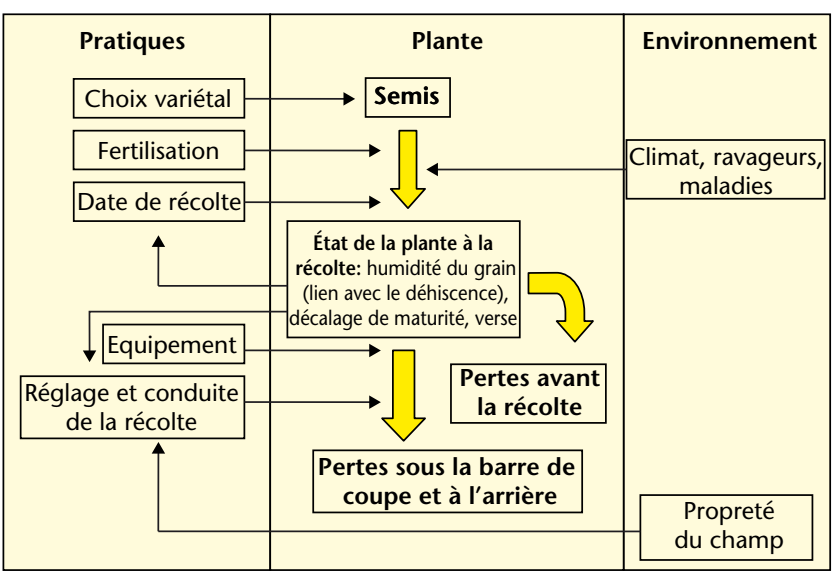

Figure 1. Facteurs de variation des pertes à la récolte, schéma d'hypothèses.

mène. L'objectif final est double : i) estimer les pertes pour améliorer notre connaissance des flux de gènes, ii) évaluer le conseil en matière de technique de récolte, pour l'améliorer le cas échéant.

\section{Matériels et méthodes}

\section{Dispositif expérimental}

Un réseau pluriannuel d'essais a été mis en place pour couvrir une gamme de climats et de milieux contrastés. Le protocole a également prévu de faire varier certaines pratiques, correspondant à des situations rencontrées en condition de production et considérées a priori comme discriminantes. Ces facteurs contrôlés ont été les suivants : variété, maturité à la récolte, matériel de récolte, à savoir modèle de moissonneuse et barre de coupe classique ou avancée (" coupe colza »). Ce dernier type de matériel est composé d'une table de coupe rallongée et de deux scies latérales (figure 2). Les expérimentations ont été mises en place lors des récoltes 2002 et 2003 sur trois sites: Dijon, Nancy et Toulouse. Une expérimentation complémentaire a été menée en 2004 à Dijon pour évaluer sur un même site l'effet d'une barre de coupe avancée. Le tableau 2 reprend l'ensemble des modalités mises en place.

Les modalités «sous-maturité » «maturité » et «sur-maturité », ont pour objectif de faire varier l'humidité du grain à la récolte, qui est dans notre schéma d'hypothèses un facteur clé pour expliquer les pertes. Pour ce faire, le protocole prévoyait de programmer les récoltes selon l'évolution de l'humidité du grain. Les dates de récolte ont été déterminées sur la base d'une observation de la culture couplée à des mesures d'humidité

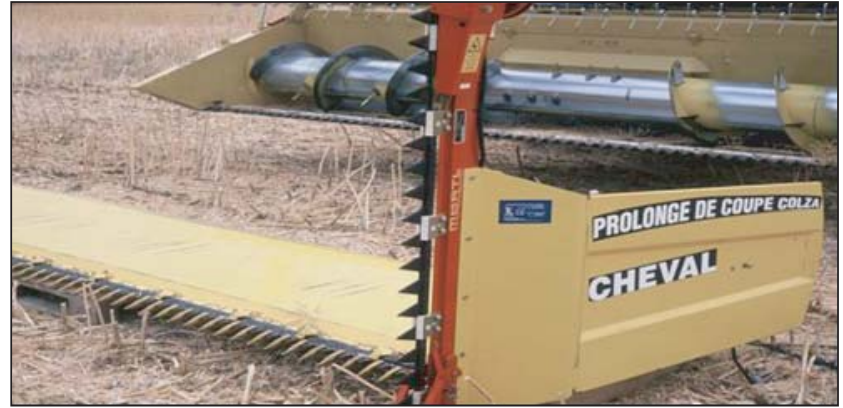

Figure 2. Barre de coupe testée à Dijon en 2004. La barre est équipée de deux scies verticales et permet une avancée de $110 \mathrm{~cm}$ par rapport à la scie de la coupe classique.

du grain, avec les objectifs suivants : « sous-maturité » supérieure à $12 \%$ d'humidité ; « maturité » entre 9 et $12 \%$, « sur-maturité » inférieure à $9 \%$. La modalité « sous-maturité » coïncidant avec de forts taux d'humidité du grain est caractéristique de certaines pratiques des producteurs du sud de la France: la partie supérieure du couvert présente une majorité de siliques mûres alors qu'une proportion non négligeable de siliques vertes reste sous-jacente. II n'est pas rare que la récolte soit entamée à ce stade par crainte de l'égrenage naturel et des orages qui menacent à cette époque de l'année.

Le réglage des machines a été laissé à l'appréciation de l'expérimentateur, dont la consigne était d'agir au mieux pour éviter les pertes quelles que soient les modalités. Le même principe a régi la conduite de la récolte, en particulier la vitesse d'avancement. II est en effet difficile de fixer ces paramètres a priori, alors qu'ils dépendent d'une adaptation fine à l'état de la culture.

Les méthodes d'estimation des pertes décrites ci-après sont, pour des raisons pratiques, difficilement compatibles avec la mise en œuvre de plans d'expérimentation factoriels. Le dispositif prévoyant un grand nombre d'essais, nous avons opté pour des expérimentations en bandes uniques pour chaque traitement expérimental. Les mesures de pertes ont été répétées trois ou quatre fois sur chaque bande à Dijon et Nancy. Les essais menés à Toulouse n'ont pas donné lieu à répétitions, un choix lié à la priorité donnée à l'estimation des pertes dans les siliques vertes non battues, une activité fortement consommatrice de temps. Le dispositif mis en place à Dijon en 2003 est illustré par la figure 3.

\section{Méthode de récupération des graines}

La méthode employée pour mesurer les pertes de graines avant la récolte et sous la barre de coupe est celle proposée par Price et al. [3], avec récupération des graines dans des bacs placés sous la culture. Les

Tableau 1. Principales références expérimentales.

\begin{tabular}{|c|c|c|c|c|c|}
\hline Référence & Lieu & Type de colza & Type de récolte & Méthode & Estimation des pertes \\
\hline Bowerman, 1984 [5] & $\begin{array}{l}\text { Boxworth et High } \\
\text { Mowthorpe (UK) }\end{array}$ & Hiver & $\begin{array}{l}\text { Andainage/dessication/ } \\
\text { directe }\end{array}$ & $\begin{array}{c}\text { Récupération dans des bacs, } \\
\text { et dans les pailles à l'arrière } \\
\text { de la machine } \\
\text { (non décrit en détails) }\end{array}$ & de 0,2 à $4 \mathrm{q} / \mathrm{ha}$ \\
\hline Price et al., 1996 [3] & UK & Hiver et printemps & Andainage/directe & Récupération dans des bacs & $\begin{array}{l}11 \% \text { en moyenne } \\
(0,9 \text { à } 24,8 \%)\end{array}$ \\
\hline $\begin{array}{l}\text { Hobson et Bruce, } \\
2002[9]\end{array}$ & Bedforshire, UK & Hiver & $\begin{array}{c}\text { Barre de coupe } \\
\text { classique/avec convoyeur }\end{array}$ & Récupération dans des bacs & $\begin{array}{c}11 \mathrm{~kg} / \mathrm{h} \text { a avant la récolte, } \\
88 \mathrm{~kg} / \mathrm{ha} \text { sous la barre avec le } \\
\text { convoyeur, } 149 \mathrm{~kg} / \mathrm{ha} \\
\text { avec la barre classique }\end{array}$ \\
\hline $\begin{array}{l}\text { Gulden et Shirtliffe, } \\
2003 \text { [4] }\end{array}$ & Ouest Canada & Printemps & Andainage & $\begin{array}{c}\text { Aspiration et tri des résidus } \\
\text { de récolte }\end{array}$ & $107 \mathrm{~kg} / \mathrm{ha}$ en moyenne \\
\hline
\end{tabular}


Tableau 2. Modalités expérimentales.

\begin{tabular}{|c|c|c|c|c|c|c|c|c|c|c|c|c|c|}
\hline \multirow{2}{*}{$\begin{array}{l}\text { Année de récolte } \\
\text { Site }\end{array}$} & \multicolumn{5}{|c|}{2002} & \multicolumn{6}{|c|}{2003} & \multicolumn{2}{|c|}{2004} \\
\hline & Dijon & \multicolumn{2}{|c|}{ Nancy } & \multicolumn{2}{|c|}{ Toulouse } & \multicolumn{2}{|c|}{ Dijon } & \multicolumn{2}{|c|}{ Nancy } & \multicolumn{2}{|c|}{ Toulouse } & \multicolumn{2}{|c|}{ Dijon } \\
\hline Barre de coupe & classique & \multicolumn{2}{|c|}{ avancée } & \multicolumn{2}{|c|}{ classique } & \multicolumn{2}{|c|}{ classique } & \multicolumn{2}{|c|}{ avancée } & \multicolumn{2}{|c|}{ classique } & \multicolumn{2}{|c|}{$\begin{array}{l}\text { classique et } \\
\text { avancée }\end{array}$} \\
\hline Moissonneuse & modèle 1 & \multicolumn{2}{|c|}{ modèle 2} & \multicolumn{2}{|c|}{ modèle 3} & \multicolumn{2}{|c|}{ modèle 1} & \multicolumn{2}{|c|}{ modèle 2} & \multicolumn{2}{|c|}{ modèle 3} & \multicolumn{2}{|c|}{ modèle 4} \\
\hline \multicolumn{14}{|l|}{ Variété } \\
\hline $\begin{array}{l}\text { Hybride restauré } 1 / 2 \text { nain } \\
\text { (DN1) }\end{array}$ & $x$ & & & $x$ & 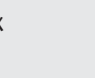 & & & & & & & & \\
\hline Hybride restauré (HR1) & $x$ & & & $x$ & 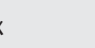 & & & & & & & & \\
\hline Lignée (L1) & $x$ & & & & & & & & & & & & \\
\hline Lignée (L2) & $x$ & & & & & & & & & & & & \\
\hline $\begin{array}{l}\text { Composite hybride-lignée } \\
\mathrm{CH} 1 \text { ) }\end{array}$ & & & & $x$ & 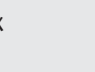 & $x$ & $x$ & & & $x$ & $x$ & & \\
\hline $\begin{array}{l}\text { Composite hybride-lignée } \\
\text { (CH2) }\end{array}$ & & & & $x$ & 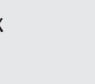 & & & & & & & & \\
\hline $\begin{array}{l}\text { Hybride restauré } 1 / 2 \text { nain } \\
\text { (DN2) }\end{array}$ & & & & & & $x$ & $x$ & $x$ & 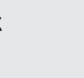 & $x$ & $x$ & & \\
\hline Lignée (L3) & & & & & & & & $x$ & 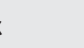 & & & & \\
\hline Lignée (L4) & \multicolumn{5}{|c|}{$x$} & & & & & & & & \\
\hline Lignée (L5) & \multirow{2}{*}{\multicolumn{5}{|c|}{$x$}} & & & & & & & & \\
\hline Hybride restauré (HR2) & & & & & & & & & & & & \multicolumn{2}{|c|}{$x$} \\
\hline \multicolumn{14}{|c|}{ Maturité (date de récolte et humidité du grain récolté) } \\
\hline Sous-maturité & & & & 26-juin & $\begin{array}{c}17- \\
21 \%\end{array}$ & & & & & 13-juin & $9-11 \%$ & & \\
\hline Maturité & 28 -juin $\quad 5-6 \%$ & 12-juil & $7-8 \%$ & 04-juil & $9-13 \%$ & 25-juin & $5-6 \%$ & 01-juil & $12 \%$ & 16-juin & $7-8 \%$ & 05-juil & $8 \%$ \\
\hline Sur-maturité & 04-juil $\quad 9-10 \%$ & & & 11-juil & $7-8 \%$ & 11-juil & $5-6 \%$ & 09-juil & $8 \%$ & 26-juin & $5-6 \%$ & & \\
\hline
\end{tabular}

dispositifs ont été adaptés selon le matériel de récolte employé et les équipements disponibles dans chaque station (tableau 3). La surface totale de réception des graines est au moins de $1 \mathrm{~m}^{2}$, sauf dans le cas de l'essai mené à Toulouse en 2002. Les bacs sont placés sous la culture au moins deux semaines avant la récolte, puis retirés juste avant celle-ci pour l'estimation des pertes par égrenage naturel, avant d'être replacés pour l'estimation des pertes sous la barre de coupe (mesure du poids brut et du nombre de graines). Pour mesurer les pertes après battage, les résidus à l'arrière des machines ont été récupérés à l'aide de bâches déroulantes ou portées. Ces résidus ont été battus pour récupérer les graines, les peser et mesurer leur poids de mille grains (PMG) brut. À Toulouse, les siliques vertes récupérées à cette étape ont été séchées en serre avant d'être battues.

La méthode a donc permis de répartir les pertes dans trois ou quatre compartiments : pertes avant la récolte ; pertes sous la barre de coupe ; pertes après battage à l'arrière de la machine ; pertes occasionnées par les siliques vertes non battues (Toulouse).

\section{Autres mesures}

Outre les pertes de graines, différentes variables ont été mesurées. Pour chaque bande : rendement grain propre et sec (GPS), et humidité du grain récolté ; pour les graines perdues récupérées dans chaque compartiment: poids de mille grains (PMG) à $0 \%$ d'humidité et taux de germination. Des observations ont été menées en culture, pour détecter certains facteurs de pertes potentiels (maladie, oiseaux, verse). Afin d'évaluer sa fiabilité comme indicateur de pertes, le nombre de repousses post-récolte a été mesuré par lancer de dix quadrats de $0,25 \mathrm{~m}^{2}$ pour chaque modalité, sur des zones distinctes de celles où ont été mesurées les pertes. Ces comptages ont été réalisés environ un mois après récolte, sans travail du sol dans l'intervalle.

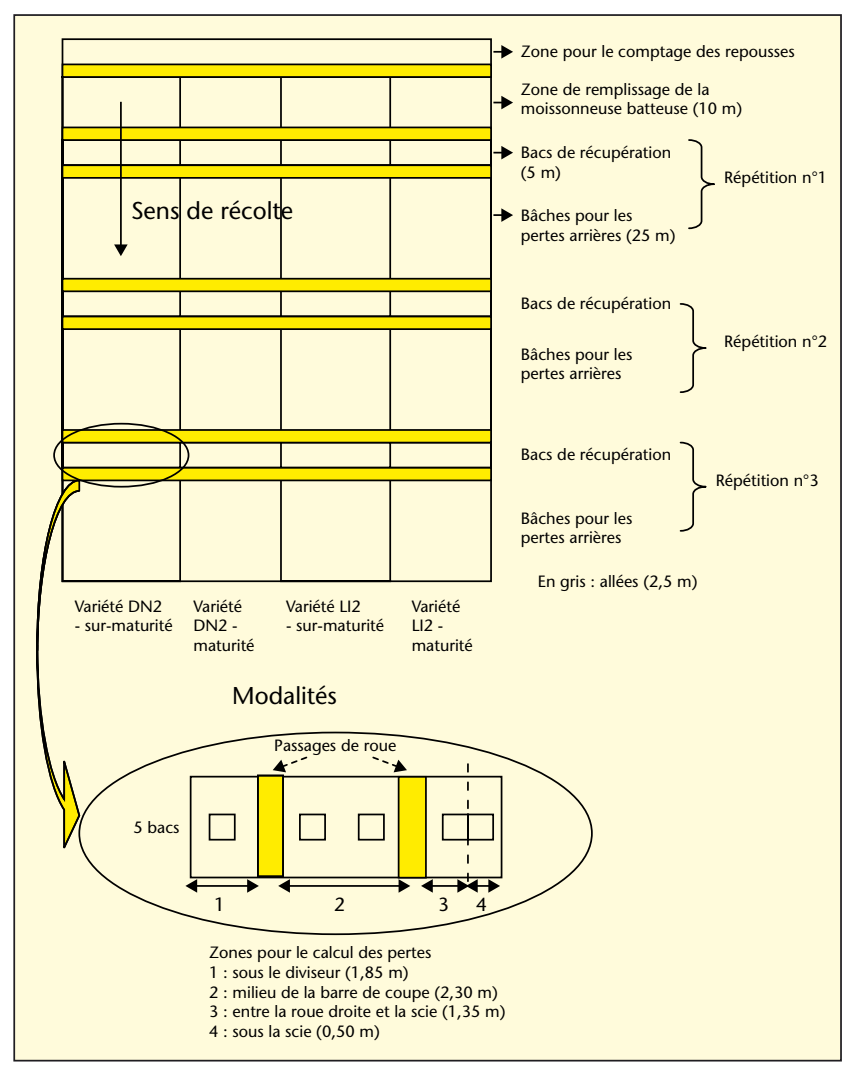

Figure 3. Plan d'expérimentation et dispositif de récupération des graines (Dijon, 2003). 
Tableau 3. Dispositifs pour l'estimation des pertes de graines.

\begin{tabular}{|c|c|c|c|c|c|c|c|}
\hline & \multicolumn{3}{|c|}{2002} & \multicolumn{3}{|c|}{2003} & \multirow{2}{*}{$\begin{array}{l}2004 \\
\text { Dijon }\end{array}$} \\
\hline & Dijon & Nancy & Toulouse & Dijon & Nancy & Toulouse & \\
\hline $\begin{array}{l}\text { Nombre de mesures } \\
\text { par bande }\end{array}$ & 3 & 3 & 1 & 3 & 3 & 1 & 4 \\
\hline Pertes avant récolte & $\begin{array}{c}3 \text { bacs * } \\
0,302 \mathrm{~m}^{2}=0,906 \mathrm{~m}^{2} \\
\text { sur } 3 \text { zones }\end{array}$ & $\begin{array}{c}5 \text { bacs * } \\
0,21 \mathrm{~m}^{2}=1,1 \mathrm{~m}^{2} \\
\text { sur } 2 \text { zones }\end{array}$ & $\begin{array}{c}6 \text { bacs dans les } \\
\text { interlignes = } \\
0,43 \mathrm{~m}^{2}\end{array}$ & $\begin{array}{c}5 \text { bacs * } \\
0,302 \mathrm{~m}^{2}=1,51 \mathrm{~m}^{2} \\
\text { sur } 4 \text { zones }\end{array}$ & $\begin{array}{c}5 \text { bacs * } \\
0,21 \mathrm{~m}^{2}=1,05 \mathrm{~m}^{2} \\
\text { sur } 2 \text { zones }\end{array}$ & 6 bacs $=1,03 \mathrm{~m}^{2}$ & 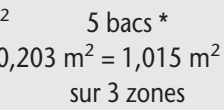 \\
\hline $\begin{array}{l}\text { Pertes sous la barre } \\
\text { de coupe }\end{array}$ & $\begin{array}{c}3 \text { bacs * } \\
0,302 \mathrm{~m}^{2}=0,906 \mathrm{~m}^{2} \\
\text { sur } 3 \text { zones }\end{array}$ & $\begin{array}{c}5 \text { bacs * } \\
0,21 \mathrm{~m}^{2}=1,1 \mathrm{~m}^{2} \\
\text { sur } 2 \text { zones }\end{array}$ & $\begin{array}{c}2 \text { bacs dans les } \\
\text { interlignes = } \\
0,49 \mathrm{~m}^{2}\end{array}$ & $\begin{array}{c}5 \text { bacs * } \\
0,302 \mathrm{~m}^{2}=1,51 \mathrm{~m}^{2} \\
\text { sur } 4 \text { zones }\end{array}$ & $\begin{array}{c}5 \text { bacs * } \\
0,21 \mathrm{~m}^{2}=1,05 \mathrm{~m}^{2} \\
\text { sur } 2 \text { zones }\end{array}$ & 6 bacs $=1,03 \mathrm{~m}^{2}$ & $\begin{array}{l}5 \text { bacs * } \\
0,203 \mathrm{~m}^{2}=1,015 \mathrm{~m}^{2} \\
\text { sur } 3 \text { zones }\end{array}$ \\
\hline Pertes à l'arrière & $\begin{array}{l}\text { bâche déroulante } \\
\qquad 60 \mathrm{~m}^{2}\end{array}$ & $\begin{array}{l}\text { bâche déroulante } \\
\qquad 54 \mathrm{~m}^{2}\end{array}$ & $\begin{array}{l}\text { bâche portée } \\
\qquad 8 \mathrm{~m}^{2}\end{array}$ & $\begin{array}{l}\text { bâche déroulante } \\
\qquad 60 \mathrm{~m}^{2}\end{array}$ & $\begin{array}{c}\text { bâche déroulante } \\
54 \mathrm{~m}^{2}\end{array}$ & $\begin{array}{l}\text { bâche portée } \\
10 \mathrm{~m}^{2}\end{array}$ & $\begin{array}{l}\text { bâche déroulante } \\
\qquad 46 \mathrm{~m}^{2}\end{array}$ \\
\hline
\end{tabular}

\section{Calcul des pertes}

Les pertes n'ont pas été estimées par une simple addition du contenu des bacs, mais après application d'une correction. Partant de l'hypothèse que les pertes obtenues sous la barre de coupe sont variables selon l'emplacement des bacs, nous avons définis deux à quatre zones suivant le type de matériel de récolte et le nombre et la position des bacs sous la barre de coupe. Les quantités de graines récupérées dans chaque bac ont été rapportées à la surface de la zone dans laquelle le bac se trouve, et en faisant l'hypothèse que chacune de ces zones subit des pertes de graines homogènes. Cette correction n'a pas été opérée à Toulouse, où les bacs ont pu être placés à équidistance les uns des autres.

\section{Résultats}

\section{Effet du taux d'humidité du grain récolté}

Les objectifs d'humidité du grain attribués à chaque modalité « maturité » n'ont pas été atteints (tableau 2). Ainsi, I'essai de Dijon en 2002 a vu la modalité « maturité » récoltée avec une humidité du grain inférieure à celle de la modalité "sur-maturité ", en raison d'un phénomène de réhumectation après une dessiccation brutale. La conjonction d'un grain $\sec (5 \%)$ et de pailles encore vertes a entrainé des conditions de récolte particulièrement difficiles qui se sont traduites par des pertes importantes sous la barre de coupe. L'humidité du grain est selon nos hypothèses initiales un des principaux facteurs de variation des pertes (figure 1). C'est donc ce facteur qui sera retenu pour les analyses qui suivent, et non le facteur « maturité ».

Le tableau 4 présente les résultats 2002 à 2004, par essai et par modalité testée, classés selon les deux principaux facteurs de variations identifiés a posteriori : le type de barre de coupe et l'humidité du grain récolté. Pour I'ensemble des points où l'humidité du grain est inférieure à $12 \%$, les pertes totales s'élèvent à $8,2 \%$ de la récolte. Au-delà de $12 \%$ d'humidité, les pertes s'élèvent rapidement (figure 4), mais ces situations correspondent uniquement aux modalités « sous-maturité » testées à Toulouse en 2002.

La répartition des pertes dans les différents compartiments dépend de I'humidité du grain récolté. Les pertes avant la récolte sont peu importantes jusqu'à $12 \%$ d'humidité, négligeables au-delà (figure 5). Elles sont parfois liées à des évènements climatiques particuliers comme ce fut le cas à Dijon en 2003 (grêle le 14 juin) avec des pertes autour de $3 \%$ de la récolte. Sous la barre de coupe, les pertes varient de près de 0 à $10 \%$ et sont d'autant plus élevées que les graines récoltées sont sèches (figure 6). Elles constituent dans ce cas la principale source de pertes totales. Les pertes à l'arrière de la machine varient de près de 0 à $10 \%$ (figure 7) sans qu'un lien puisse être établi avec l'humidité du grain. Les résultats obtenus à Toulouse montrent que les pertes causées par les siliques vertes non battues peuvent être extrêmement importantes quand l'humidité du grain dépasse $17 \%$, mais des risques de pertes existent dès $9 \%$ (figure 8). Ces résultats indiquent les risques pris à récolter trop sec ou trop humide. Un grain sec n'induit pas nécessairement d'augmentation de pertes avant récolte, bien qu'il puisse entraîner une sensibilité accrue à des évènements climatiques exceptionnels. Par contre, il provoque des pertes importantes sous la barre de coupe. Un grain humide augmente quant à lui les pertes à l'arrière de la moissonneuse dues aux siliques non battues.

\section{Effet du matériel de récolte}

Les pertes obtenues à Nancy en 2002 et 2003 avec coupe avancée sont cinq fois inférieures à celles obtenues à Dijon et Toulouse avec coupe classique pour des rendements et des taux d'humidité équivalents. L'expérimentation réalisée à Dijon en 2004 visait à vérifier cet effet du matériel de récolte indépendamment du site. Sous la barre de coupe, les pertes moyennes rapportées à l'hectare sur la modalité « coupe classique » sont de $2,64( \pm 0,73)$ q contre $0,22( \pm 0,12)$ q pour celles mesurées sur la modalité « coupe avancée » (tableau 5). Il apparaît que la réduction des pertes a lieu principalement sous la barre de coupe, en particulier dans la zone médiane entre les scies latérales. Les pertes à l'arrière de la moissonneuse, $0,16 \mathrm{q} / \mathrm{ha}( \pm 0,03)$ pour la coupe classique contre 0,13 $q /$ ha $( \pm 0,04)$ pour la coupe « avancée ", ne semblent pas être affectées par le type de matériel. Par contre, les résultats obtenus sur les trois années d'expérimentation suggèrent que ces pertes arrière dépendent du modèle de moissonneuse (figure 7).

\section{Les pertes de graines dépendent-elles des variétés?}

Le dispositif ne nous a pas permis d'appréhender un effet variétal, car ce facteur n'est pas indépendant d'autres facteurs, notamment l'humidité du grain à la récolte, qui varient eux-mêmes selon les conditions de récolte de chaque essai.

\section{Caractéristiques des graines tombées au sol}

Le PMG des graines perdues avant récolte est inférieur au PGM des graines perdues sous la barre de coupe (figure 9). Par contre, celui-ci ne diffère pas significativement du PMG des graines perdues derrière la moissonneuse. Les tests de germination montrent qu'au moins $90 \%$ graines sont viables, y compris dans les siliques vertes non battues.

\section{Nombre de repousses et estimation des pertes}

La méthode d'estimation du nombre de repousses après récolte s'est avérée peu fiable pour les fortes densités (supérieures à 8000 repousses $/ \mathrm{m}^{2}$ ), les plantules ne pouvant plus être dénombrées individuellement. Ceci pourrait expliquer pourquoi les valeurs obtenues à 
Tableau 4. Résultats obtenus sur les expérimentations menées en 2002 et 2003, classés selon les facteurs type de barre de coupe et humidité du grain récolté.

\begin{tabular}{|c|c|c|c|c|c|c|c|c|c|c|}
\hline \multirow[t]{2}{*}{ Année } & \multirow[t]{2}{*}{ Variété } & \multirow[t]{2}{*}{ Lieu } & \multirow{2}{*}{$\begin{array}{c}\text { Humidité du } \\
\text { grain } \\
\text { récolté (\%) }\end{array}$} & \multirow{2}{*}{$\begin{array}{l}\text { Rendement } \\
\text { GPS* (q/ha) }\end{array}$} & \multirow{2}{*}{$\begin{array}{c}\text { Pertes } \\
\text { graines à } \\
0 \% \\
\text { humidité } \\
\text { (q/ha) }\end{array}$} & \multicolumn{5}{|c|}{ Pertes par rapport au rendement GPS (\%) } \\
\hline & & & & & & Totales & $\begin{array}{l}\text { Avant la } \\
\text { récolte }\end{array}$ & $\begin{array}{c}\text { Sous la } \\
\text { barre de } \\
\text { coupe }\end{array}$ & $\begin{array}{c}\text { À l'arrière } \\
\text { de la } \\
\text { machine }\end{array}$ & $\begin{array}{c}\text { Sur siliques } \\
\text { non battues }\end{array}$ \\
\hline \multicolumn{11}{|c|}{ coupe « colza » : barre de coupe avancée + scies latérales } \\
\hline 2002 & L5 & Nancy & 7,2 & 27,84 & 0,73 & 2,62 & 0,39 & 2,14 & 0,08 & - \\
\hline 2004 & HR2 & Dijon & 7,6 & 28,68 & - & - & - & 0,76 & 0,44 & - \\
\hline 2002 & L4 & Nancy & 7,9 & 27,63 & 0,63 & 2,26 & 0,39 & 1,67 & 0,20 & - \\
\hline 2003 & DN2 & Nancy & 8,1 & 22,70 & 0,47 & 2,08 & 0,76 & 0,72 & 0,60 & - \\
\hline 2003 & L3 & Nancy & 8,1 & 25,20 & 0,37 & 1,46 & 0,67 & 0,46 & 0,34 & - \\
\hline 2003 & DN2 & Nancy & 12,1 & 22,80 & 0,37 & 1,61 & 0,08 & 0,21 & 1,32 & - \\
\hline 2003 & L3 & Nancy & 12,1 & 23,80 & 0,34 & 1,42 & 0,02 & 0,28 & 1,12 & - \\
\hline \multicolumn{11}{|c|}{ coupe « classique " } \\
\hline 2002 & DN1 & Dijon & 4,9 & 25,90 & 3,18 & 12,26 & 0,03 & 7,76 & 4,47 & - \\
\hline 2002 & HR1 & Dijon & 5,0 & 27,80 & 3,46 & 12,46 & 0,06 & 8,76 & 3,64 & - \\
\hline 2002 & L1 & Dijon & 5,1 & 24,60 & - & - & 0,03 & 5,64 & - & - \\
\hline 2003 & L2 & Dijon & 5,2 & 28,60 & 3,01 & 10,52 & 2,61 & 4,39 & 3,53 & - \\
\hline 2003 & $\mathrm{CH} 1$ & Toulouse & 5,4 & 23,80 & 3,36 & 14,11 & 1,03 & 4,17 & 8,88 & 0,02 \\
\hline 2003 & L2 & Dijon & 5,6 & 26,10 & 3,75 & 14,35 & 3,07 & 6,57 & 4,71 & - \\
\hline 2003 & DN2 & Dijon & 5,6 & 21,70 & 2,72 & 12,55 & 2,55 & 3,24 & 6,76 & - \\
\hline 2003 & DN2 & Dijon & 5,7 & 20,50 & 2,51 & 12,24 & 2,95 & 4,69 & 4,60 & - \\
\hline 2003 & DN2 & Toulouse & 5,9 & 21,90 & 3,17 & 14,49 & 0,39 & 3,54 & 9,65 & 0,92 \\
\hline 2002 & L2 & Dijon & 5,9 & 30,80 & - & - & 0,03 & 5,03 & - & - \\
\hline 2003 & DN2 & Toulouse & 7,0 & 18,50 & 1,94 & 10,47 & 0,86 & 5,68 & 3,30 & 0,62 \\
\hline 2002 & $\mathrm{CH} 1$ & Toulouse & 7,0 & 35,31 & 3,40 & 9,63 & 1,18 & 5,36 & 2,99 & 0,09 \\
\hline 2004 & HR2 & Dijon & 7,6 & 26,01 & - & - & - & 10,10 & 0,63 & - \\
\hline 2002 & DN1 & Toulouse & 7,6 & 36,90 & 3,84 & 10,41 & 0,42 & 2,41 & 7,39 & 0,19 \\
\hline 2003 & $\mathrm{CH} 1$ & Toulouse & 7,7 & 23,80 & 1,97 & 8,27 & 0,31 & 5,63 & 2,30 & 0,03 \\
\hline 2002 & HR1 & Toulouse & 8,1 & 36,25 & 2,16 & 5,95 & 2,46 & 1,60 & 1,72 & 0,18 \\
\hline 2002 & $\mathrm{CH} 2$ & Toulouse & 8,1 & 32,35 & 1,83 & 5,67 & 0,51 & 1,42 & 2,92 & 0,82 \\
\hline 2002 & HR1 & Dijon & 8,8 & 27,90 & 2,22 & 7,97 & 3,33 & 3,35 & 1,29 & - \\
\hline 2002 & L1 & Dijon & 8,9 & 23,60 & 1,92 & 8,15 & 1,60 & 3,99 & 2,56 & - \\
\hline 2003 & DN2 & Toulouse & 9,0 & 20,10 & 3,93 & 19,53 & 0,51 & 2,11 & 3,20 & 13,70 \\
\hline 2002 & DN1 & Dijon & 9,0 & 26,50 & 1,61 & 6,07 & 1,02 & 2,24 & 2,80 & \\
\hline 2002 & HR1 & Toulouse & 9,4 & 36,12 & - & - & - & 2,31 & 2,41 & 0,82 \\
\hline 2002 & DN1 & Toulouse & 9,5 & 36,23 & - & - & - & 3,59 & 4,02 & 3,63 \\
\hline 2002 & L2 & Dijon & 9,9 & 27,90 & 1,77 & 6,33 & 1,94 & 2,54 & 1,85 & - \\
\hline 2003 & $\mathrm{CH} 1$ & Toulouse & 11,4 & 25,00 & 3,16 & 12,66 & 0,46 & 1,65 & 1,25 & 9,30 \\
\hline 2002 & $\mathrm{CH} 2$ & Toulouse & 13,0 & 34,47 & - & - & - & 2,17 & 5,82 & 4,03 \\
\hline 2002 & $\mathrm{CH} 1$ & Toulouse & 17,2 & 33,43 & 14,39 & 43,04 & 0,00 & 1,74 & 8,15 & 33,16 \\
\hline 2002 & HR1 & Toulouse & 18,1 & 30,67 & 10,49 & 34,21 & 0,00 & 0,58 & 3,80 & 29,84 \\
\hline 2002 & $\mathrm{CH} 2$ & Toulouse & 21,2 & 24,12 & 20,30 & 84,16 & 0,00 & 0,69 & 4,15 & 79,32 \\
\hline 2002 & DN1 & Toulouse & 21,3 & 24,00 & 15,89 & 66,23 & 0,00 & 0,39 & 6,73 & 59,11 \\
\hline \multicolumn{4}{|c|}{ Pertes moyennes pour les humidités $<12 \%$} & 26,96 & 2,22 & 8,25 & & & & \\
\hline
\end{tabular}

* Grain propre et sec ; - données non disponibles ; en italique : rendement estimé.

Toulouse en 2002 sont largement supérieures au nombre de graines tombées au sol, calculées à partir des mesures de perte.

Les densités de repousses dépendent étroitement des conditions climatiques avant et après la récolte. Les densités les plus faibles obtenues à Dijon et Nancy en 2003 peuvent être mises en relation avec des conditions sèches pendant cette période. À l'inverse, une pluie bien placée suffit à provoquer une levée massive, comme à Toulouse en 2002. La figure 10 montre le peu de fiabilité de la relation entre le nombre de repousses après la récolte et le nombre de graines perdues.

\section{Discussion}

Intérêt et limite de la méthode d'évaluation des pertes

La méthode de récupération des graines, particulièrement lourde pour les pertes à l'arrière des engins de récolte, n'autorise pas la mise en œuvre aisée d'expérimentations factorielles classiques. Néanmoins, dans le cadre de dispositifs en bandes, un certain nombre de répétitions des mesures semble indispensable pour effectuer des estimations correctes : le coefficient de variation observé à Dijon en 2004 sur les pertes totales de 


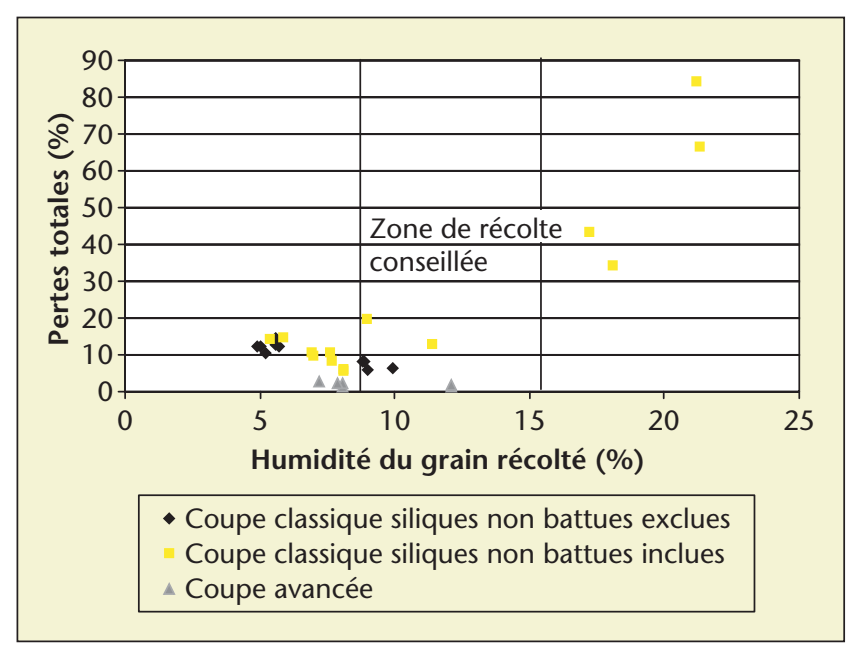

Figure 4. Pertes totales par rapport au rendement en fonction de l'humidité du grain récolté (2002 et 2003).

chaque modalité est de $25 \%$. La méthode a permis de répartir les pertes de graines en trois ou quatre compartiments, ce qui permet de mieux apprécier le rôle exact de telle pratique de récolte ou choix d'équipement. Des pertes parfois importantes ont été mises en évidence à l'arrière de la machine. L'importance des pertes liées aux siliques vertes non battues pour les humidités du grain élevées était inattendue.

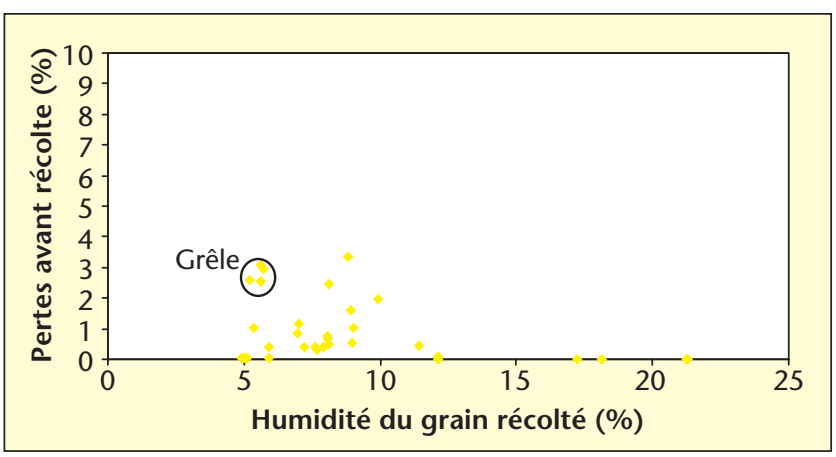

Figure 5. Pertes avant la récolte exprimées en pourcentage du rendement, selon I'humidité du grain récolté $(2002,2003)$.

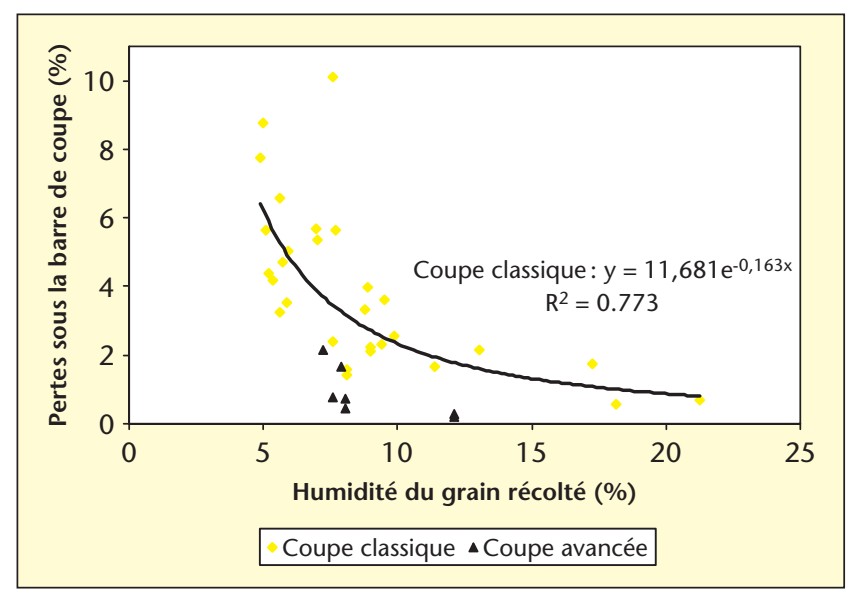

Figure 6. Pertes sous la barre de coupe exprimées en pourcentage du rendement, selon I'humidité du grain récolté (2002 à 2004).

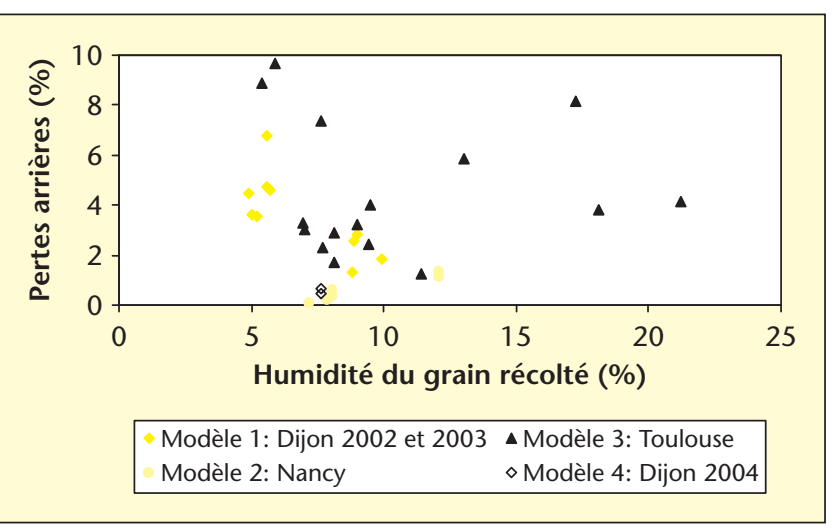

Figure 7. Pertes à l'arrière de la batteuse exprimées en pourcentage du rendement, selon l'humidité du grain récolté (2002 à 2004). Chaque série de données correspond à un modèle particulier de moissonneuse batteuse.

Les pertes avant la récolte sont connues pour dépendre des conditions climatiques. Ce lien n'a pas été clairement mis en évidence, car les expérimentations n'ont pas connu de conditions exceptionnelles, comme des orages violents pouvant causer la perte de la quasi-totalité de la récolte. Aucun phénomène de verse n'a non plus été constaté.

Les résultats présentés ne prennent pas en compte l'hétérogénéité de la maturité mais seulement des humidités moyennes du grain récolté. Les relations entre humidité et pertes manquent par conséquent de précision. II n'a par exemple pas été possible de déterminer dans quelle mesure les pertes dans les siliques vertes non battues observées à $9 \%$ d'humidité peuvent être attribuées à la fraction de grains dont l'humidité est supérieure à cette moyenne. En d'autres termes, il n'a pas été possible de vérifier si l'hétérogénéité de la maturité autour de cette valeur seuil de $9 \%$ favorise ce type de pertes. Toutefois les résultats suggèrent que I'hétérogénéité de la maturité a tendance à augmenter les pertes totales. En effet, la non-linéarité de la relation entre pertes sous la barre de coupe et humidité du grain augmente en principe la contribution des graines les plus sèches, et par conséquent les pertes totales. L'hétérogénéité de la maturité accroîtrait également la sensibilité des pertes avant la récolte. Enfin, I'utilisation du nombre de repousses post-récolte comme indicateur des pertes de graines donne de mauvais résultats, car les conditions climatiques post-récolte compliquent l'interprétation des résultats : un été sec suffit à empêcher toute levée, mais dans le cas contraire, la densité de repousses est telle que son estimation devient difficilement praticable.

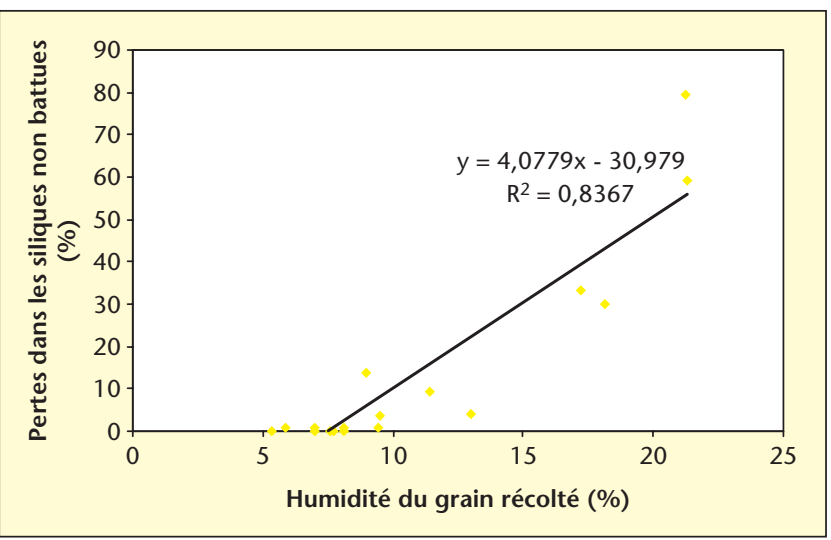

Figure 8. pertes dans les siliques non battues exprimées en pourcentage du rendement, selon I'humidité du grain récolté $(2002,2003)$. 
Tableau 5. Pertes sous la barre de coupe, en q/ha à 0\% d'humidité (Dijon, 2004).

\begin{tabular}{|lccccc|}
\hline & & \multicolumn{2}{c|}{ Coupe classique, 4 répétitions } & \multicolumn{2}{c|}{ Coupe avancée, 4 répétitions } \\
\cline { 2 - 6 } Bac & Superficie* & Moyenne & Écart-type & Moyenne & Écart-type \\
\hline Gauche & $2 / 18$ & 0,93 & 0,23 & 0,26 & 0,10 \\
Médian & $7 / 27$ & 4,41 & 0,82 & 0,12 & 0,07 \\
Médian & $7 / 27$ & 2,47 & 1,57 & 0,17 & 0,10 \\
Médian & $7 / 27$ & 2,88 & 0,68 & 0,24 & 0,26 \\
Droite & $2 / 18$ & 0,51 & 0,34 & 0,31 & 0,11 \\
Barre de coupe & & $\mathbf{2 . 6 4}^{* *}$ & $\mathbf{0 , 7 3}$ & $\mathbf{0 . 2 2}^{* *}$ & $\mathbf{0 , 1 2}$ \\
\hline
\end{tabular}

* représente le facteur correctif par lequel a été multiplié chaque mesure, pour rendre compte de la surface que représente chaque bac dans la parcelle. ** différence significative selon un test $\mathrm{T}(\mathrm{p}<0,005)$.

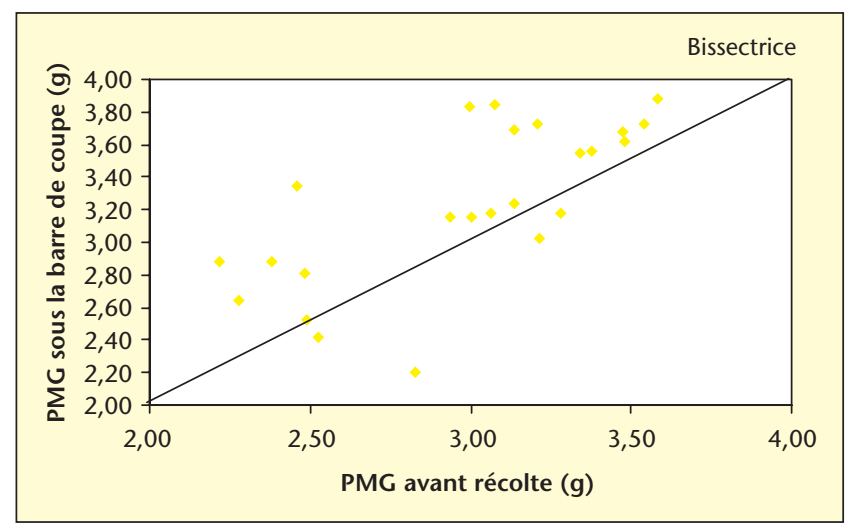

Figure 9. Comparaison des poids de mille grains (PMG) à $0 \%$ d'humidité des grains perdus avant la récolte et sous la barre de coupe. Les pertes sont significativement différentes selon un test $T(p<0,001)$.

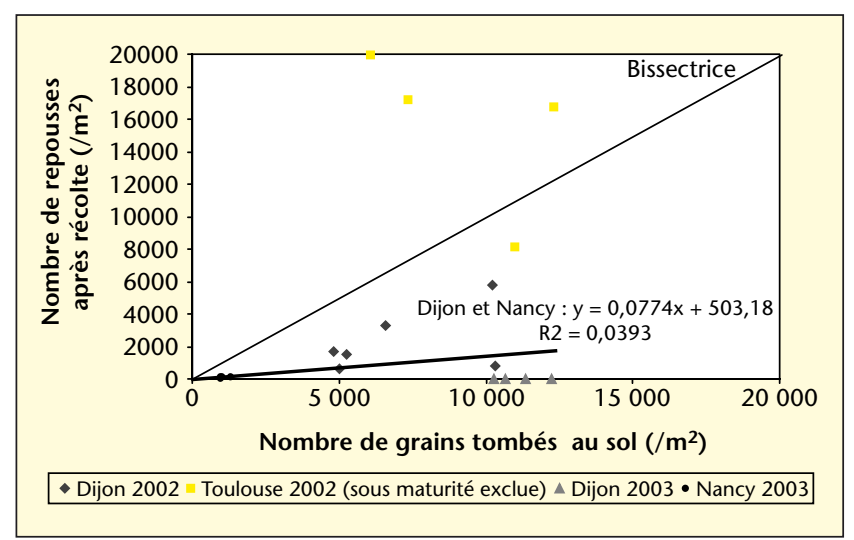

Figure 10. Nombre de repousses après la récolte en lien avec le nombre total de graines perdues. Les mesures réalisées à Toulouse 2002 consistent en des estimations visuelles.

\section{Voies pour améliorer le conseil}

Il est conseillé aux agriculteurs de récolter dans une fourchette d'humidité du grain de 8 à $15 \%$, pour atteindre des objectifs de facilité de récolte (paille suffisamment sèche), de réduction des pertes, ainsi que de qualité technologique des graines. Les résultats obtenus semblent cohérents avec ce message technique, car cet intervalle correspond à des pertes plutôt faibles (figure 4), dont une part importante survient sous la barre de coupe. Mais il convient de souligner que les conditions climatiques $n^{\prime}$ ont pas permis l'obtention de nombreux points aux alentours de $15 \%$ d'humidité pour mieux étayer notre affirmation. D'autre part, des risques de pertes dans les siliques vertes non battues sont relevés à partir de $9 \%$ d'humidité.

Dans la gamme d'humidité conseillée, l'adoption d'une barre de coupe avancée semble donc une voie intéressante pour limiter les pertes. Dans les conditions de l'expérimentation menée à Dijon en 2004, le gain peut être estimé à $60 € /$ ha (colza payé au prix de $21 € / q)$ ). En considérant les rendements et les prix 2004, ainsi qu'une durée de vie de l'équipement de 10 ans, l'investissement de $6000 €$ se voit justifié à partir d'une superficie récoltée en colza de 10 ha par an. Mais ce chiffre reste indicatif, car il gomme la variabilité due à l'état de la culture, la conduite de la récolte et le type de matériel. En outre, il ne prend pas en compte le fait qu'une coupe avancée autorise une récolte plus facile et plus rapide. L'enquête annuelle 2005 réalisée par le Cetiom sur les itinéraires techniques en colza montre que le taux d'équipement en coupe avancée est de $40 \%$ en France avec toutefois de fortes disparités régionales [10]. Les matériels proposés sont divers, avec des rallonges de coupe variant de 50 à $95 \mathrm{~cm}$, ce qui suggère des réductions de pertes variables.

La pratique consistant à anticiper la récolte par crainte de l'égrenage naturel n'a pas porté les fruits escomptés dans le contexte de l'expérimentation. Au contraire, alors que les pertes avant la récolte restent faibles, on constate une explosion des pertes dans les siliques vertes non battues.

Le conseil technique actuel insiste sur I'humidité des graines comme critère de déclenchement du chantier de récolte. Or cet indicateur est difficile à apprécier et peut évoluer rapidement, et pas seulement dans le sens d'une diminution au fil du temps! L'agriculteur a parfois l'opportunité de faire réaliser des analyses par sa coopérative, mais la maturité optimale se juge souvent d'après des critères visuels (couleur du couvert, des graines). Pour traduire les normes d'humidité en pratique, l'agriculteur observe, tient compte du scénario climatique de l'année. La décision de récolter dépend aussi d'autres facteurs liés à l'organisation des chantiers. L'hétérogénéité de la maturité complique parfois cette décision. L'observation d'un couvert hétérogène peut provoquer la récolte alors que les pailles sont encore vertes, ce qui ralentit le chantier et pourrait augmenter les risques de pertes.

Les pertes à l'arrière de la moissonneuse semblent incompressibles dans la gamme d'humidité conseillée pour un matériel de récolte donné. Leur niveau correspond à un compromis effectué par l'agriculteur entre deux exigences contradictoires : l'éjection des petites graines par un soufflage énergique doit être évitée, mais dans le même temps le réglage doit garantir un taux d'impureté inférieur à la norme de $2 \%$. II paraît difficile d'établir des règles précises pour réaliser ces arbitrages, qui dépendraient autant des caractéristiques du matériel, que de l'état du colza et de la propreté du champ. Par contre, des marges de manœuvre pourraient éventuellement être trouvées au niveau de la conception des matériels de récolte. 


\section{Impact sur les flux de gènes}

Les mesures de PMG correspondent à un flux entrant de 1038 à 13014 graines $/ \mathrm{m}^{2}$ pour les humidités du grain inférieures à $15 \%$, avec une moyenne de 8 277, et dont la grande majorité sont viables. Ces résultats permettront d'améliorer le paramétrage de modèle de flux de gènes comme GENESYS-colza [11], où l'estimation des pertes est laissée à la libre appréciation de l'utilisateur. L'analyse de sensibilité de ce modèle a en effet montré l'importance du stock semencier initial, qui influence les résultats de simulation pendant cinq à six ans [12]. Or, la constitution du stock semencier est en étroite relation avec le flux de graines entrant. Dans I'optique de contribuer à l'amélioration des modèles de flux de gènes, on peut envisager la constitution d'une grille de risque de pertes, au sein de laquelle on pourra situer les agriculteurs d'une région donnée en fonction de leurs pratiques de récolte. Cependant, nous avons vu le rôle important joué par le climat, avec la possibilité d'évènements catastrophiques, ou bien des scénarios inhabituels pouvant contrecarrer les prévisions des agriculteurs. Les résultats obtenus peuvent contribuer à établir une plage de variations aléatoires des pertes autour d'une moyenne.

Remerciements. Ces travaux ont été financés en partie par la société SERASEM.

\section{RÉFÉRENCES}

1. GRUBER S, PEKRUN C, CLAUPEIN W. Population dynamics of volunteer oilseed rape (Brassica napus L.) affected by tillage. Eur / Agron 2004; 30 : 351-61.

2. LUTMAN PJW. The occurrence and persistence of volunteer oilseed rape (Brassica napus). Asp Appl Biol 1993 ; 35 : 29-36.
3. PRICE JS, HOBSON RN, NEALE MA, BRUCE DM. Seed losses in commercial harvesting of oilseed rape. J Agric Engng Res 1996 ; 65 : 183-91.

4. GULDEN RH, SHIRTLIFFE SJ. Harvest losses of canola (Brassica napus) cause large seedbank inputs. Weed Sci $2003 ;$ 51(1) : 83-6.

5. BOWERMAN P. Comparison of harvesting methods of oilseed rape. Asp Appl Biol $1984 ; 6$ : 157-65.

6. JAWORSKI CA, PHATAK SC. Canola Seed Yield in Relation to Harvest Methods. Proceedings of the Second National Symposium. New Crops: Exploration Research and Commercialization 06-09 octobre 1991.

7. KOLEK L, VASAK J, FAMERA M, KAVKA M. The effect of time of harvest on losses and quality of production of rape, wheat and barley. Rostlinna Vyrobo 1997 ; 43(6) : 275-82 ; (en Tchèque).

8. RAWA T, WIERZBICKI K. Effect of some factors on the rape grain losses during direct harvesting. Roczniki Nauk Rolniczych/C-Technika Rolnicza 1993 ; 79(4) : 23-32 ; (en Polonais).

9. HOBSON RN, BRUCE DM. Seed Loss when Cutting a Standing Crop of Oilseed Rape with Two types of Combine Harvester Header. Biosystems Engineering $2002 ; 81(3)$ : 181-286.

10. WAGNER D, QUERE L, REAU R. Enquête colza 2005, synthèse annuelle. CETIOM, 2006.

11. COLBACH N, CLERMONT-DAUPHIN C, MEYNARD JM. GeneSys : A model of the influence of cropping system on gene escape from herbicide tolerant rapeseed crops to rape volunteers. I. Temporal evolution of a population of rapeseed volunteers in a field. Agric Ecosyst Environ $2001 ; 83$ : 235-53.

12. COLBACH N, MOLINARI N, CLERMONT-DAUPHIN C. Sensitivity analysis for a model simulating demography and genotype evolutions with time. Application to GENESYS modelling gene flow between rape seed varieties and volunteers. Ecol Modell $2004 ; 179$ : 91-113. 